PROCEEDINGS OF THE

AMERICAN MATHEMATICAL SOCIETY

Volume 138, Number 10, October 2010, Pages 3481-3494

S 0002-9939(10)10376-1

Article electronically published on May 5, 2010

\title{
FACTORING NEWPARTS OF JACOBIANS OF CERTAIN MODULAR CURVES
}

\author{
M. RAM MURTY AND KANEENIKA SINHA
}

(Communicated by Wen-Ching Winnie Li)

\begin{abstract}
We prove a conjecture of Yamauchi which states that the level $N$ for which the new part of $J_{0}(N)$ is $\mathbb{Q}$-isogenous to a product of elliptic curves is bounded. We also state and partially prove a higher-dimensional analogue of Yamauchi's conjecture. In order to prove the above results, we derive a formula for the trace of Hecke operators acting on spaces $S^{n e w}(N, k)$ of newforms of weight $k$ and level $N$. We use this trace formula to study the equidistribution of eigenvalues of Hecke operators on these spaces. For any $d \geq 1$, we estimate the number of normalized newforms of fixed weight and level, whose Fourier coefficients generate a number field of degree less than or equal to $d$.
\end{abstract}

\section{INTRODUCTION}

For a positive integer $N$, let $\Gamma_{0}(N)$ denote the set of all matrices in $S L_{2}(\mathbb{Z})$ such that $N$ divides the lower left entry and let $X_{0}(N)$ denote the quotient of the extended upper half plane by the action of $\Gamma_{0}(N)$. It can be viewed as an algebraic curve defined over $\mathbb{Q}$. Let $J_{0}(N)$ denote the Jacobian variety of $X_{0}(N)$. For a positive divisor $M$ of $N$, let $N e w_{M}$ denote the set of normalized newforms of weight 2 with respect to $\Gamma_{0}(M)$ and, for each $f(z)=\sum_{n=1}^{\infty} a_{n}(f) e^{2 \pi i n z} \in N e w_{M}$, let $K_{f}=\mathbb{Q}\left(\left\{a_{n}(f)_{n \geq 1}\right\}\right)$. By the work of Shimura [21, one can associate to each $f \in N e w_{M}$ an abelian variety quotient $A_{f}$ of $J_{0}(N)$ such that if $f$ and $f^{\prime} \in$ New $M$ are Galois conjugates, then $A_{f}$ is $\mathbb{Q}$-isogenous to $A_{f^{\prime}}$. (We denote this as $A_{f} \sim \mathbb{Q} A_{f^{\prime}}$.) Later, Ribet [17] showed that each $A_{f}$ is $\mathbb{Q}$-simple. Ribet also observed (see Proposition 3.2 of [2]) that the converse is true; that is, for $f$ and $f^{\prime} \in N e w_{M}, A_{f} \sim_{\mathbb{Q}} A_{f^{\prime}}$ only if $f$ and $f^{\prime}$ are Galois conjugates. Thus, by the work of Ribet and Shimura, we have the decomposition

$$
J_{0}(N) \sim_{\mathbb{Q}} \bigoplus_{M \mid N} \bigoplus_{f \in N e w_{M} / G_{\mathbb{Q}}} A_{f}^{n_{f}},
$$

where $G_{\mathbb{Q}}=\operatorname{Gal}(\overline{\mathbb{Q}} / \mathbb{Q}), n_{f}$ denotes the number of positive divisors of $N / M$, and the dimension of $A_{f}$ is equal to $\left[K_{f}: \mathbb{Q}\right]$. For a fixed positive integer $d$, the problem of determining all levels $N$ for which all $\mathbb{Q}$-simple factors of $J_{0}(N)$ are of dimension less

Received by the editors August 4, 2009 and, in revised form, January 7, 2010.

2010 Mathematics Subject Classification. Primary 11F11, 11F25, 11F30, 11G10, $11 \mathrm{G} 18$.

Key words and phrases. Traces of Hecke operators, Fourier coefficients of modular cusp forms, modular curves, Jacobian varieties.

The first author was partially supported by an NSERC grant.

The second author was partially supported by a PIMS fellowship.

(C)2010 American Mathematical Society Reverts to public domain 28 years from publication 
than or equal to $d$ has been well investigated. By a result of Serre (see Theorem 7 of [20]), we know that there are only finitely many levels $N$ for which this happens. In [5], Cohen listed all the odd $N$ 's for the case $d=1$, that is, all the $N$ 's such that all $\mathbb{Q}$-simple factors of $J_{0}(N)$ are elliptic curves. Building on Cohen's work, Yamauchi 22] listed all the $N$ 's (even and odd) such that all $\mathbb{Q}$-simple factors of $J_{0}(N)$ are elliptic curves. In [15], we made Serre's result effective; that is, for any $d \geq 1$, we found an effectively computable constant $B(d)$, which depends only on $d$ such that if all $\mathbb{Q}$-simple factors of $J_{0}(N)$ are of dimension $\leq d$, then $N \leq B(d)$.

In this article, our focus is on the newpart of $J_{0}(N)$, denoted as $J_{0}^{n e w}(N)$, for which we have the decomposition

$$
J_{0}^{\text {new }}(N) \sim_{\mathbb{Q}} \bigoplus_{f \in N e w_{N} / G_{\mathbb{Q}}} A_{f} .
$$

In 22, Yamauchi conjectured that if $J_{0}^{\text {new }}(N)$ is $\mathbb{Q}$-isogenous to a product of elliptic curves, then $N$ is bounded above by 1800 . In section 5 of this paper, we essentially prove this conjecture in the following theorem:

Theorem 1. If $J_{0}^{n e w}(N)$ is $\mathbb{Q}$-isogenous to a product of elliptic curves, then $N$ is bounded above by an absolute and effectively computable constant.

We can formulate a higher dimensional analogue of Yamauchi's conjecture, as follows:

Conjecture 2. For any $d \geq 1$, there are only finitely many positive integers $N$ such that $J_{0}^{\text {new }}(N)$ is $\mathbb{Q}$-isogenous to a product of $\mathbb{Q}$-simple abelian varieties of dimension less than or equal to $d$.

Let $p$ be a prime number. In 19, Royer proved that for any sufficiently large number $N$ coprime to $p, J_{0}^{\text {new }}(N)$ has a $\mathbb{Q}$-simple factor of dimension $\gg \sqrt{\log \log N}$, where the implied constant depends on $p$. This immediately implies that for any $d \geq 1$, there are only finitely many $N$ 's not divisible by $p$ such that all $\mathbb{Q}$-simple factors of $J_{0}^{\text {new }}(N)$ are of dimension less than or equal to $d$. This result for the case $p=2$ was also independently proved by Lim [12]. However, neither Royer's nor Lim's results are effective. In the direction of Conjecture 2, we prove the following theorem in section 5 :

Theorem 3. Let $p$ be a fixed prime. For any integer $d \geq 1$, if $N$ is coprime to $p$ and $J_{0}^{\text {new }}(N)$ is isogenous to a product of $\mathbb{Q}$-simple abelian varieties of dimension less than or equal to $d$, then $N$ is bounded above by a constant $B(p, d)$ that depends only on $p$ and $d$. More precisely,

$$
\log 2 N \leq 311206 d^{2} 2^{4 d^{2}} p^{d^{2} / 2} \log p .
$$

Remark 4. The correct way to interpret the value of Royer's result and Theorem 3 is that if we fix a prime $p$, then Conjecture 2 holds for positive integers $N$ coprime to $p$. The advantage of Theorem 3 over Royer's result is that it clearly reveals the dependence of the bound for $N$ on $p$ and $d$. This explicit bound enables us to address Yamauchi's conjecture (for the case $d=1$ ) for all $N$. If we take $p=2$, Theorem 3 leads to an effective upper bound for all odd $N$ 's in Conjecture 2 that is, an effective version of Lim's result. The constant in Theorem 1 obtained by our current methods turns out to be much larger than the one conjectured by Yamauchi. We relegate the sharpening of this constant to future work. 
In the next section, we state some results that lead to Theorems 1 and 3 and are also of independent interest.

\section{Preliminaries}

Let $S(N, k)$ be the space of cusp forms of weight $k$ ( $k \geq 2$ is an even integer) with respect to $\Gamma_{0}(N)$ and, for any integer $n \geq 1$, let $T_{n}(N, k)$ be the $n$-th Hecke operator acting on $S(N, k)$. Let $s(N, k)$ be the dimension of $S(N, k)$. Let $p$ be a fixed prime and let $\left(k_{\lambda}, N_{\lambda}\right)$ be a sequence of pairs of positive integers with $N_{\lambda}+k_{\lambda} \rightarrow \infty$ provided that $p$ does not divide $N_{\lambda}$ and $k_{\lambda}$ is even. By the theorem of Deligne proving the Ramanujan-Petersson inequality, we know that the eigenvalues of $T_{p}\left(N_{\lambda}, k_{\lambda}\right)$ lie in the interval

$$
\left[-2 p^{\frac{k_{\lambda}-1}{2}}, 2 p^{\frac{k_{\lambda}-1}{2}}\right] .
$$

In his celebrated 1997 paper [20], Serre proved that with $N_{\lambda}+k_{\lambda} \rightarrow \infty$ as above, the family of eigenvalues of the normalized $p$-th Hecke operator

$$
T_{p}^{\prime}\left(N_{\lambda}, k_{\lambda}\right)=\frac{T_{p}\left(N_{\lambda}, k_{\lambda}\right)}{p^{\left(k_{\lambda}-1\right) / 2}}
$$

is equidistributed in the interval $[-2,2]$ with respect to the measure

$$
\mu_{p}= \begin{cases}\frac{p+1}{\pi} \frac{\left(1-x^{2} / 4\right)^{1 / 2}}{\left(p^{1 / 2}+p^{-1 / 2}\right)^{2}-x^{2}} d x & \text { if } x \in[-2,2] \\ 0 & \text { otherwise. }\end{cases}
$$

In [15, we proved the following effective version of Serre's equidistribution theorem (see [15], p. 701). Let $N$ be a positive integer and let $p$ be a prime not dividing $N$. Let $\left\{\lambda_{p, i}\right\}_{1 \leq i \leq s(N, k)}$ denote the eigenvalues of $T_{p}(N, k)$. For an interval $[\alpha, \beta] \subset$ $[-2,2]$ and for any positive integer $M \geq 1$,

$$
\begin{gathered}
\quad\left|\left\{\# i: \frac{\lambda_{p, i}}{p^{\frac{k-1}{2}}} \in[\alpha, \beta]\right\}-s(N, k) \int_{\alpha}^{\beta} \mu_{p}(x)\right| \\
\ll \frac{s(N, k)}{M+1}+\left(p^{3 M / 2} 2^{\nu(N)} \sigma_{0}(N) \sqrt{N} \log p\right) M \log M,
\end{gathered}
$$

where $\nu(N)$ denotes the number of prime divisors of $N$ and $\sigma_{0}(N)$ denotes the number of positive divisors of $N$. This effective version of Serre's theorem has several applications. Most notably, in [15] we estimate, for a given $d$ and prime $p$ not dividing $N$, the number of eigenvalues of $T_{p}$ of degree less than or equal to $d$. We then determine an effectively computable constant $B_{d}$ such that if $J_{0}(N)$ is isogenous to a product of $\mathbb{Q}$-simple abelian varieties of dimensions less than or equal to $d$, then $N \leq B_{d}$.

In this paper, we restrict our attention to the eigenvalues of $T_{p}$ acting on $S^{n e w}(N, k)$, the space of newforms of weight $k$ and level $N$. We denote the dimension of $S^{\text {new }}(N, k)$ by $s^{\text {new }}(N, k)$. In [20, Serre also shows that the eigenvalues of $T_{p}^{\prime}$ acting on $S^{\text {new }}\left(N_{\lambda}, k_{\lambda}\right)$ are equidistributed with respect to $\mu_{p}$. We obtain precise error terms in the effective equidistribution of eigenvalues of $T_{p}$ acting on $S^{n e w}(N, k)$ and apply our effective results to study the factorization of $J_{0}^{\text {new }}(N)$.

We first compute a formula for the trace of $T_{n}$ acting on $S^{n e w}(N, k)$, which we denote as $T_{n}^{n e w}(N, k)$. This is an important ingredient in obtaining effective equidistribution results. Although a formula for $s^{n e w}(N, k)$ is now known by the work of Martin [13], the trace formula for $T_{n}^{n e w}(N, k)$ in closed form has so far 
not been computed. It is therefore worthwhile to fill this gap in the literature. In section 3 , we prove the following theorem:

Theorem 5. Let $n$ be a positive integer coprime to $N$. The trace of the Hecke operator $T_{n}^{\text {new }}(N, k)$ is given by

$$
\begin{gathered}
\begin{cases}n^{(k / 2-1)} \cdot \frac{k-1}{12} N B_{1}(N) & \text { if } n \text { is a square, } \\
0 & \text { otherwise }\end{cases} \\
-\frac{1}{2} \sum_{t \in \mathbb{Z}, t^{2}<4 n} \frac{\varrho^{k-1}-\bar{\varrho}^{k-1}}{\varrho-\bar{\varrho}} \sum_{f} h_{w}\left(\frac{t^{2}-4 n}{f^{2}}\right) B_{2}(N)_{f} \\
-\sum_{\substack{d \mid n \\
0<d \leq \sqrt{n}}}^{\prime} d^{k-1} B_{3}(N)_{d}+ \begin{cases}\mu(N) \sum_{t \mid n} t & \text { if } k=2, \\
0 & \text { otherwise }\end{cases}
\end{gathered}
$$

where

- $B_{1}(N)$ is a multiplicative function such that for a prime power $p^{r}$,

$$
B_{1}\left(p^{r}\right)= \begin{cases}1-\frac{1}{p} & \text { if } r=1, \\ 1-\frac{1}{p}-\frac{1}{p^{2}} & \text { if } r=2, \\ \left(1-\frac{1}{p}\right)\left(1-\frac{1}{p^{2}}\right) & \text { if } r \geq 3 .\end{cases}
$$

- $\varrho$ and $\bar{\varrho}$ are the zeroes of the polynomial $x^{2}-t x+n$.

- The inner sum in the second term runs over all positive divisors $f$ of $t^{2}-4 n$ such that $\left(t^{2}-4 n\right) / f^{2} \in \mathbb{Z}$ is congruent to 0 or $1 \bmod 4$.

- $h_{w}(\Delta)$ is the class number of the imaginary quadratic order of discriminant $\Delta$ divided by 2 (resp. 3) if the discriminant is -4 (resp. -3).

- For a positive integer $f, B_{2}(N)_{f}$ is a multiplicative function of $N$ such that

$$
B_{2}(p)_{f}= \begin{cases}p-1 & \text { if } p \mid f \\ -1+\left(\frac{t^{2}-4 n}{p}\right) & \text { otherwise }\end{cases}
$$

where $\left(\frac{*}{p}\right)$ denotes the Legendre symbol. If $N=p^{r}$ for some $r \geq 2$ and $p^{b}|| f$, then

$$
B_{2}\left(p^{r}\right)_{f}=\sum_{i=r-2}^{r} \sigma_{0}^{-1}\left(p^{r-i}\right) \frac{\psi\left(p^{i}\right)}{\psi\left(p^{i-\min \{i, b\}}\right)} M\left(t, n, p^{i+\min \{i, b\}}\right),
$$

where

$$
\psi(N)=N \prod_{p \mid N}\left(1+\frac{1}{p}\right),
$$

$\sigma_{0}^{-1}(N)$ denotes the Dirichlet inverse of $\sigma_{0}(N)$ and $M\left(t, n, p^{i+\min \{i, b\}}\right)$ denotes the number of elements of $\left(\mathbb{Z} / p^{i} \mathbb{Z}\right)^{*}$ which lift to solutions of $x^{2}-$ $t x+n \equiv 0 \bmod \left(p^{i+\min \{i, b\}}\right)$.

- The prime on the summation in the third term of $\operatorname{Tr} T_{n}^{n e w}(N, k)$ indicates that if there is a contribution from the term $d=\sqrt{n}$, it should be multiplied by $1 / 2$. 
- $B_{3}(N)_{d}$ is a multiplicative function of $N$ such that for a prime power $p^{r}$,

$$
B_{3}\left(p^{r}\right)_{d}= \begin{cases}-\phi\left(p^{\frac{r-2}{2}}\right) & \text { if } r \text { is even and } p^{\frac{r-2}{2}} \|\left(\frac{n}{d}-d\right), \\ \phi\left(p^{\frac{r}{2}}\right)-\phi\left(p^{\frac{r-2}{2}}\right) & \text { if } r \text { is even and } p^{\frac{r}{2}} \mid\left(\frac{n}{d}-d\right), \\ 0 & \text { otherwise. }\end{cases}
$$

Henceforth, let $p$ be a prime not dividing $N$. For any closed interval $[\alpha, \beta] \subset$ $[-2,2]$, let $E^{\text {new }}(p, N, k,[\alpha, \beta])$ denote the number of eigenvalues (counted with multiplicity) of the normalized Hecke operator $T_{p}^{\prime \text { new }}(N, k)$ lying in the interval $[\alpha, \beta]$. Also, for any $x \in[-2,2]$, let us define $\theta_{x} \in[0, \pi]$ such that $2 \cos \theta_{x}=x$. In section 4 , we prove the following theorems:

Theorem 6. For any interval $[\alpha, \beta] \subset[-2,2]$ and for any positive integer $M$, we have

$$
\begin{gathered}
\left|E^{\text {new }}(p, N, k,[\alpha, \beta])-s^{\text {new }}(N, k) \int_{\alpha}^{\beta} \mu_{p}\right| \leq 4 \frac{s^{\text {new }}(N, k)}{M+1}+19 p^{2 M+1} 4^{\nu(N)} \\
+2 \sum_{1 \leq m \leq M} \min \left(\frac{\theta_{\alpha}-\theta_{\beta}}{2 \pi}, \frac{1}{m \pi}\right)\left(10 p^{2 m+1} 4^{\nu(N)}+\left|s^{\text {new }}(N, k)-N B_{1}(N) \frac{k-1}{12}\right| \mathcal{C}_{m}\right),
\end{gathered}
$$

where, for every $m \geq 1$,

$$
\mathcal{C}_{m}= \begin{cases}0 & \text { if } m \text { is odd } \\ \frac{1}{p} & \text { if } m=2 \\ \frac{1}{p^{m-2}}-\frac{1}{p^{m}} & \text { if } m \geq 4 \text { is even } .\end{cases}
$$

Theorem 7. Let $\left\{a_{p, i}\right\}, 1 \leq i \leq s^{\text {new }}(N, k)$, denote the family of eigenvalues of $T_{p}^{n e w}(N, k)$. For any $\alpha \in\left[-2 p^{(k-1) / 2}, 2 p^{(k-1) / 2}\right]$ and for any $c>3$,

$$
\#\left\{1 \leq i \leq s^{n e w}(N, k): a_{p, i}=\alpha\right\} \leq 8 c s^{n e w}(N, k) \frac{\log p}{\log k N}+237\left(\frac{6 c}{(c-3) e}\right)^{2} \frac{k N}{(\log k N)^{2}} .
$$

Theorem 8. For any positive integer d, let

$$
s^{\text {new }}(N, k, p)_{d}=\#\left\{1 \leq i \leq s^{\text {new }}(N, k):\left[\mathbb{Q}\left(a_{p, i}\right): \mathbb{Q}\right] \leq d\right\} .
$$

For any $c>3$, we have

$$
s^{\text {new }}(N, k, p)_{d} \leq \mathcal{C}_{d, p, k}\left\{8 c s^{\text {new }}(N, k) \frac{\log p}{\log k N}+237\left(\frac{6 c}{(c-3) e}\right)^{2} \frac{k N}{(\log k N)^{2}}\right\},
$$

where

$$
C_{d, p, k}=d^{2} \prod_{i=1}^{d}\left(2\left(\begin{array}{c}
d \\
i
\end{array}\right)\left(2 p^{\frac{k-1}{2}}\right)^{i}+1\right) .
$$

Remark 9 . The above bound is non-trivial only if we can choose $p$ sufficiently small so that $C_{d, p, k} \ll(\log k N)^{a}$ for some $a<1$. 


\section{Eichler-Selberg trace formula And modifications}

The Eichler-Selberg trace formula describes the trace of $T_{n}$ acting on $S(N, k)$. Following the presentation of this formula in [11, p. 370], for every integer $n \geq 1$, $\operatorname{Tr} T_{n}(N, k)=\sum_{i=1}^{4} A_{i}(n, N, k)$, where $A_{i}$ 's are as follows:

$$
\begin{gathered}
A_{1}(n, N, k)=\frac{k-1}{12} \psi(N) \begin{cases}n^{(k / 2-1)} & \text { if } n \text { is a square, } \\
0 & \text { otherwise. }\end{cases} \\
A_{2}(n, N, k)=-\frac{1}{2} \sum_{t \in \mathbb{Z}, t^{2}<4 n} \frac{\varrho^{k-1}-\bar{\varrho}^{k-1}}{\varrho-\bar{\varrho}} \sum_{f} h_{w}\left(\frac{t^{2}-4 n}{f^{2}}\right) \mu(t, f, n) .
\end{gathered}
$$

Here, $\varrho, \bar{\varrho}, f$ and $h_{w}(\Delta)$ are as in Theorem 5 and

$$
\mu(t, f, n)=\frac{\psi(N)}{\psi\left(\frac{N}{N_{f}}\right)} M\left(t, n, N N_{f}\right),
$$

where $N_{f}=\operatorname{gcd}(N, f)$ and $M\left(t, n, N N_{f}\right)$ denotes the number of elements of $(\mathbb{Z} / N \mathbb{Z})^{*}$ which lift to solutions of $x^{2}-t x+n \equiv 0 \bmod N N_{f}$.

$$
A_{3}(n, N, k)=-\sum_{\substack{d \mid n, 0<d \leq \sqrt{n}}}^{\prime} d^{k-1} F(N)_{d},
$$

where $F(N)_{d}$ is a multiplicative function of $N$ defined as

$$
F(N)_{d}=\sum_{\substack{c\left|N \\ \operatorname{gcd}\left(c, \frac{N}{c}\right)\right| \frac{n}{d}-d}} \phi\left(\operatorname{gcd}\left(c, \frac{N}{c}\right)\right)
$$

The prime on the summation defining $A_{3}(n, N, k)$, just as in Theorem 1 , indicates that if there is a contribution from the term $d=\sqrt{n}$, it should be multiplied by $\frac{1}{2}$.

$$
A_{4}(n, N, k)= \begin{cases}\sum_{t \mid n, t>0} t & \text { if } k=2 \\ 0 & \text { otherwise }\end{cases}
$$

By the Atkin-Lehner decomposition [1], we know that

$$
S(N, k)=\bigoplus_{d \mid N} \bigoplus_{a \mid\left(\frac{N}{d}\right)} i_{a, d}\left(S^{n e w}(d, k)\right)
$$

where, for positive integers $a$ and $d$ such that $a d \mid N, i_{a, d}$ denotes the embedding $f(z) \mapsto f(a z)$ of $S(d, k)$ into $S(N, k)$. Thus,

$$
s(N, k)=\sum_{d \mid N} \sigma_{0}\left(\frac{N}{d}\right) s^{n e w}(d, k) .
$$

From this, we deduce that

$$
s^{n e w}(N, k)=\sum_{d \mid N} \sigma_{0}^{-1}\left(\frac{N}{d}\right) s(d, k),
$$


where $\sigma_{0}^{-1}(m)$ denotes the inverse of $\sigma_{0}(m)$ with respect to Dirichlet convolution; that is, $\sigma_{0}^{-1}(N)$ is a multiplicative function defined as follows on prime powers:

$$
\sigma_{0}^{-1}\left(p^{r}\right)= \begin{cases}1 & \text { if } r=0 \text { or } 2 \\ -2 & \text { if } r=1 \\ 0 & \text { if } r>2\end{cases}
$$

This idea was utilised by Martin (13, Theorem 1) to derive a closed-form and computationally efficient formula for $s^{\text {new }}(N, k)$ from previously known formulae for $s(N, k)$. This inversion technique can also be used to derive an explicit formula for $\operatorname{Tr} T_{n}^{n e w}(N, k)$, using the Eichler-Selberg trace formula for $\operatorname{Tr} T_{n}(N, k)$, provided $n$ is coprime to $N$. We observe that if $(n, N)=1$ and $f(z) \in S^{n e w}(d, k)$ for some $d \mid N$, then $T_{n}$ has the same eigenvalue on $f(z)$ as it does on $i_{a, d}(f)$. Thus, by the Atkin-Lehner decomposition (1), we get that

$$
\operatorname{Tr} T_{n}(N, k)=\sum_{d \mid N} \sigma_{0}\left(\frac{N}{d}\right) \operatorname{Tr} T_{n}^{\text {new }}(d, k) .
$$

Thus,

$$
\operatorname{Tr} T_{n}^{\text {new }}(N, k)=\sum_{d \mid N} \sigma_{0}^{-1}\left(\frac{N}{d}\right) \operatorname{Tr} T_{n}(d, k) .
$$

The following can be easily verified by checking them at prime powers:

$$
\begin{gathered}
\sum_{d \mid N} \sigma_{0}^{-1}(N / d) \psi(d)=N B_{1}(N), \quad \sum_{d \mid N} \sigma_{0}^{-1}(N / d) \mu(t, f, d)=B_{2}(N)_{f}, \\
\sum_{r \mid N} \sigma_{0}^{-1}(N / r) F(r)_{d}=B_{3}(N)_{d} \quad \text { and } \quad \sum_{d \mid N} \sigma_{0}^{-1}(N / d)=\mu(N) .
\end{gathered}
$$

Combining the above facts, we are able to obtain $\sum_{d \mid N} \sigma_{0}^{-1}(N / d) A_{i}(n, d, k)$ for each $i$. This proves Theorem 5 .

Remark 10. The idea of performing Möbius inversion on equation (2) was also utilised by Hamer [9] to obtain the trace of $T_{n}^{*}(N, k)$, the Hecke operator $T_{n}$ acting on

$$
S^{*}(N, k)=\bigoplus_{d \mid N} S^{n e w}(N, k)
$$

provided $(n, N)=1$. She observes that equation (2) can also be written as

$$
\operatorname{Tr} T_{n}(N, k)=\sum_{d \mid N} \operatorname{Tr} T_{n}^{*}(d, k)
$$

She then obtains a trace formula for $T_{n}^{*}(N, k)$ for squarefree $N$ by Möbius inversion.

Remark 11. For $n=1$, the trace of $T_{n}^{n e w}(N, k)$ is equal to $s^{n e w}(N, k)$. Thus, Proposition 5 for $n=1$ gives the same formula as Theorem 1 of [13. Since $B_{3}(N)_{1}=0$ if $N$ is not a square, we immediately deduce that

$$
\left|s^{\text {new }}(N, k)-N B_{1}(N) \frac{k-1}{12}\right| \leq \begin{cases}\frac{\sqrt{N}}{2}+\frac{7}{12} 2^{\nu(N)}+1 & \text { if } N \text { is a square } \\ \frac{7}{12} 2^{\nu(N)}+1 & \text { otherwise. }\end{cases}
$$

We now state two results which will help us obtain explicit bounds on the terms of the trace formula. 
Proposition 12. For a positive integer $N>0$, let

$$
H(N)=\sum_{d^{2} \mid N} h_{w}\left(\frac{-N}{d^{2}}\right)
$$

where the sum runs over all positive divisors $d$ of $N$ such that $-N / d^{2} \in \mathbb{Z}$ is congruent to 0 or $1(\bmod 4)$. Then,

$$
\sum_{t^{2}<4 n} H\left(4 n-t^{2}\right)=2 \sigma_{1}(n)-\lambda(n)+\frac{1}{6}
$$

where

$$
\lambda(n)=\sum_{d \mid n} \min \left(d, \frac{n}{d}\right) \text { and } \sigma_{1}(n)=\sum_{\substack{d \mid n \\ d>0}} d .
$$

Proof. The above recursion formula is due to Kronecker and Gierster (see 6], pp. 108 and 127). It was also proved by Eichler (see [7, equation (6)) by interpreting the numbers $H(N)$ in terms of the number of fixed points of Hecke's correspondences on the Riemann surface $X_{0}(2)$. We have stated this formula as it appears in Theorem 5.3.8 of [4].

Proposition 13. Suppose $a$ and $b$ are integers such that $a^{2}-4 b \neq 0$. Given an integer $K$, the number of solutions $\bmod K$ of the congruence $x^{2}-a x+b \equiv 0 \bmod K$ is less than or equal to $2^{\nu(K)} \sqrt{\left|a^{2}-4 b\right|}$.

Proof. This proposition forms the content of Huxley's paper [10].

From Propositions 12 and 13, we deduce the following:

Proposition 14. Let $p$ be a prime not dividing $N$. Then, for any $m>0$,

$\mid \operatorname{Tr} T_{p^{m}}^{\prime n e w}(N, k)-N B_{1}(N) \frac{k-1}{12}\left\{\begin{array}{ll}p^{-m / 2} & \text { if } m \text { is even } \\ 0 & \text { otherwise }\end{array} \mid \leq 8 p^{2 m+1} 4^{\nu(N)}+p^{m / 2+1}\right.$.

Proof. Inserting Proposition 13 in $B_{2}(N)_{f}$, we deduce that

$$
\left|B_{2}(N)_{f}\right| \leq 4^{\nu(N)} \psi(f) \sqrt{4 n-t^{2}}
$$

Combining this estimate for $B_{2}(N)$ with Proposition 12, we get that

$$
\left|\frac{1}{2} \sum_{t \in \mathbb{Z}, t^{2}<4 n} \frac{\varrho^{k-1}-\bar{\varrho}^{k-1}}{\varrho-\bar{\varrho}} \sum_{f} h_{w}\left(\frac{t^{2}-4 n}{f^{2}}\right) B_{2}(N)\right| \leq 8 n^{\frac{k+1}{2}} 4^{\nu(N)} \sigma_{0}(n) .
$$

We also observe that for any $n>1$,

$$
\sum_{\substack{d \mid n \\ 0<d \leq \sqrt{n}}}^{\prime} d^{k-1} B_{3}(N)_{d} \leq \sum_{\substack{d \mid n \\ 0<d \leq \sqrt{n}}} d^{k}
$$

In particular, taking $n=p^{m}, m \geq 1$ and dividing by $\left(p^{m}\right)^{\frac{k-1}{2}}$, we prove Proposition 14 


\section{EFFECTIVE EQUIDISTRIBUTION RESULTS}

Let us consider the compact set $[0,1]$ of $\mathbb{R}$. Let $A_{1}, A_{2}, \cdots$ be a sequence of finite nonempty multisets of $[0,1]$ with $\# A_{n} \rightarrow \infty$ as $n \rightarrow \infty$, where $\# A_{n}$ denotes the cardinality of $A_{n}$. We say that $\left\{A_{n}\right\}$ is equidistributed with respect to a measure $\mu$ if for every $A \subseteq[0,1]$,

$$
\lim _{n \rightarrow \infty} \frac{\#\left\{t \in A_{n}: t \in A\right\}}{\# A_{n}}=\mu(A) .
$$

Let $e(x):=e^{2 \pi i x}$. Suppose that the Weyl limits of this sequence,

$$
c_{m}=\lim _{n \rightarrow \infty} \frac{1}{\# A_{n}} \sum_{t \in A_{n}} e(m t),
$$

exist for every $m \in \mathbb{Z}$ and $\sum_{m=1}^{N}\left|c_{m}\right|^{2}=\mathrm{o}(N)$. Then, by a generalization of the Wiener-Schoenberg theorem (see [14], Theorem 11.3.3, p. 181) the measure $\mu$ is given by $F(-x) d x$, where $F(x)=\sum_{m=-\infty}^{\infty} c_{m} e(m x)$. Let $\|\mu\|$ be the supremum of $|F(x)|$ for $x \in[0,1]$. In [15], we proved the following all-purpose effective equidistribution theorem:

Theorem 15. For any $I=[a, b] \subseteq[0,1]$, let $N_{I}(V):=\#\left\{t \in A_{V}: t \in I\right\}$ and let $D_{I, V}(\mu):=\left|N_{I}(V)-V \mu(I)\right|$. Then,

$D_{I, V}(\mu) \leq \frac{V\|\mu\|}{M+1}+\sum_{\substack{m=-M \\ m \neq 0}}^{M}\left(\frac{1}{M+1}+\min \left(b-a, \frac{1}{\pi|m|}\right)\right)\left|\sum_{n=1}^{V} e\left(m x_{n}\right)-V c_{m}\right|$,

if $V$ and $M$ are natural numbers.

For a prime $p$ not dividing $N$, let $\left\{\frac{a_{p, i}}{p^{\frac{k-1}{2}}}\right\}, 1 \leq i \leq s^{\text {new }}(N, k)$, denote the family of eigenvalues of $T_{p}^{\prime \text { new }}(N, k)$. For each $i$, choose $\theta_{p, i} \in[0, \pi]$ such that

$$
\frac{a_{p, i}}{p^{\frac{k-1}{2}}}=2 \cos \theta_{p, i} \text {. }
$$

We study the distribution of the sequence $\pm \frac{\theta_{p, i}}{2 \pi}(\bmod 1)\left(1 \leq i \leq s^{n e w}(N, k)\right)$. The Weyl limits, in this case, are

$$
\begin{gathered}
c_{m}=\lim _{\substack{N+k \rightarrow \infty \\
(+, N)=1 \\
k \text { even }}} \frac{1}{2 s^{\text {new }}(N, k)} \sum_{i=1}^{s^{\text {new }}(N, k)}\left\{e\left(\frac{m \theta_{p, i}}{2 \pi}\right)+e\left(-\frac{m \theta_{p, i}}{2 \pi}\right)\right\} \\
=\lim _{\substack{N+k \rightarrow \infty \\
(, N)=1 \\
k \text { even }}} \frac{1}{2 s^{\text {new }}(N, k)} \sum_{i=1}^{s^{\text {new }}(N, k)} 2 \cos \left(m \theta_{p, i}\right) .
\end{gathered}
$$

For $m=1$,

$$
\sum_{i} 2 \cos \left(m \theta_{p, i}\right)=\operatorname{Tr} T_{p}^{\prime n e w}(N, k)
$$

and for $m \geq 2$,

$$
\sum_{i} 2 \cos \left(m \theta_{p, i}\right)=\operatorname{Tr} T_{p^{m}}^{\prime}{ }^{\text {new }}(N, k)-\operatorname{Tr} T_{p^{m-2}}^{\prime}{ }^{\text {new }}(N, k) .
$$


Thus, from Proposition 14 and equation (3), we deduce that the Weyl limits $c_{m}$ 's are given by $c_{0}=1, c_{m}=0$ for $m$ odd; and for $m$ even,

$$
c_{m}=\frac{1}{2}\left(\frac{1}{p^{|m| / 2}}-\frac{1}{p^{(|m|-2) / 2}}\right) .
$$

We also observe that for any $m \geq 1$,

$$
\begin{gathered}
\sum_{i} 2 \cos m \theta_{p, i}-2 s^{\text {new }}(N, k) c_{m} \\
= \begin{cases}\operatorname{Tr} T_{p}^{\prime \text { new }}(N, k) & \text { if } m=1, \\
\operatorname{Tr} T_{p^{2}}^{\prime \text { new }}(N, k)-\frac{s^{\text {new }}(N, k)}{p} & \text { if } m=2, \\
\operatorname{Tr} T_{p^{m}}^{\prime \text { new }}(N, k)-\operatorname{Tr} T_{p^{m-2}}^{\prime}{ }^{\text {new }}(N, k)+\mathcal{C}_{m} s^{\text {new }}(N, k) & \text { if } m \geq 3,\end{cases}
\end{gathered}
$$

where the $\mathcal{C}_{m}$ 's are as defined in Theorem 6 .

By substituting the above information in Theorem 15 , we get that for any $[\alpha, \beta] \subset$ $[-2,2]$ and for any $M \geq 1$,

$$
\begin{gathered}
\left|E^{\text {new }}(p, N, k,[\alpha, \beta])-s^{\text {new }}(N, k) \int_{\alpha}^{\beta} \mu_{p}\right| \leq \frac{2 s^{\text {new }}(N, k)}{M+1} \\
+\sum_{1 \leq|m| \leq M}\left(\frac{1}{M+1}+\min \left(\frac{\theta_{\alpha}-\theta_{\beta}}{2 \pi}, \frac{1}{\pi|m|}\right)\right)\left|\sum_{i} 2 \cos m \theta_{p, i}-2 s^{\text {new }}(N, k) c_{m}\right| \\
\leq \frac{2 s^{\text {new }}(N, k)}{M+1}+19 p^{2 M+1} 4^{\nu(N)}+\frac{2}{M+1}\left(\frac{2}{p}-\frac{1}{p^{M / 2}}\right)\left|s^{\text {new }}(N, k)-N B_{1}(N) \frac{k-1}{12}\right| \\
+2 \sum_{m=1}^{M} \min \left(\frac{\theta_{\alpha}-\theta_{\beta}}{2 \pi}, \frac{1}{m \pi}\right)\left(11 p^{2 m+1} 4^{\nu(N)}+\left|s^{\text {new }}(N, k)-N B_{1}(N) \frac{k-1}{12}\right|\right) \mathcal{C}_{m} .
\end{gathered}
$$

This proves Theorem 6. Thus, for a fixed $\alpha \in\left[-2 p^{(k-1) / 2}, 2 p^{(k-1) / 2}\right]$ and for any $M \geq 1$,

$$
\#\left\{1 \leq i \leq s^{n e w}(N, k): a_{p, i}=\alpha\right\} \leq 4 \frac{s^{n e w}(N, k)}{M+1}+19 p^{2 M+1} 4^{\nu(N)} .
$$

By a result of Ramanujan (see equation (200) of [16]), we know that

$$
\sigma_{0}(N) \leq 8\left(\frac{3 N}{35}\right)^{1 / 3}
$$

Since $2^{\nu(N)} \leq \sigma_{0}(N)$, we deduce that

$$
4^{\nu(N)} \leq 64\left(\frac{3 N}{35}\right)^{2 / 3}
$$

For any $c>3$ we choose

$$
M+1=\left[\frac{\frac{1}{c} \log k N}{2 \log p}\right] .
$$

By elementary calculus, we know that $\log x \leq x^{a} / a e$, for any $a>0$ and $x \geq 1$. Thus,

$$
(k N)^{\frac{2}{3}+\frac{1}{c}} \leq\left(\frac{6 c}{(c-3) e}\right)^{2} \frac{k N}{(\log k N)^{2}} .
$$


Thus, for any $c>3$,

$$
\#\left\{1 \leq i \leq s^{n e w}(N, k): a_{p, i}=\alpha\right\} \leq 8 c s^{n e w}(N, k) \frac{\log p}{\log k N}+237\left(\frac{6 c}{(c-3) e}\right)^{2} \frac{k N}{(\log k N)^{2}} \text {. }
$$

This proves Theorem [7. We now recall the following proposition from [15]:

Proposition 16. For a positive integer $d$ and a real number $K>0$, the number of algebraic integers $\alpha$ of degree $d$ and $H(\alpha) \leq K$ is at most

$$
\prod_{i=1}^{d}\left(2\left(\begin{array}{c}
d \\
i
\end{array}\right) K^{i}+1\right)
$$

where $H(\alpha)$ is the maximum of the absolute values of all conjugates of $\alpha$.

Proof. This is Proposition 30 of [15].

If $\alpha$ is an eigenvalue of $T_{p}^{n e w}(N, k)$, then the absolute values of all conjugates of $\alpha$ are bounded above by $2 p^{(k-1) / 2}$. Thus, taking $K=2 p^{\frac{k-1}{2}}$ in Proposition 16, we deduce that if $\alpha$ is an eigenvalue of $T_{p}^{n e w}(N, k)$ such that $[\mathbb{Q}(\alpha): \mathbb{Q}] \leq d$, then $\alpha$ can take at most

$$
C_{d, p, k}=d^{2} \prod_{i=1}^{d}\left(2\left(\begin{array}{c}
d \\
i
\end{array}\right)\left(2 p^{\frac{k-1}{2}}\right)^{i}+1\right)
$$

values. This, combined with Theorem 7 , proves Theorem 8

By the work of Atkin and Lehner [1] $S^{\text {new }}(N, k)$ has a unique basis, say, $\left\{f_{i}\right\}_{1 \leq i \leq s^{\text {new }}(N, k)}$, consisting of normalized newforms. For any such form $f_{i}(z)=$ $\sum_{n=1}^{\infty} a_{n}\left(f_{i}\right) e^{2 \pi i n z}$, let

$$
K_{i}=\mathbb{Q}\left(\left\{a_{n}\left(f_{i}\right)\right\}_{n \geq 1}\right) .
$$

$K_{i}$ is a finite extension of $\mathbb{Q}$. For any integer $d \geq 1$, we define

$$
s^{\text {new }}(N, k)_{d}=\#\left\{1 \leq i \leq s^{\text {new }}(N, k):\left[K_{i}: \mathbb{Q}\right]=d\right\} .
$$

Clearly, for any prime $p$ not dividing $N$,

$$
\sum_{r=1}^{d} s^{n e w}(N, k)_{r} \leq s^{n e w}(N, k, p)_{d} .
$$

In particular, taking $c=20$ in Theorem 8 , we deduce that for every $d \geq 1$,

$$
\sum_{r=1}^{d} s^{n e w}(N, k)_{r} \leq C_{d, p, k}\left(160 s^{n e w}(N, k) \frac{\log p}{\log k N}+1617 \frac{k N}{(\log k N)^{2}}\right) .
$$

We observe that

$$
\mathcal{C}_{d, p, k} \leq d^{2} 2^{4 d^{2}}\left(p^{\frac{k-1}{2}}\right)^{\frac{d(d+1)}{2}},
$$

and for $N \geq 2$ (see, for example, Theorem $3.1(\mathrm{~g})$ of $[3]$ ),

$$
\phi(N) \geq \frac{N \log 2}{\log 2 N} .
$$

Moreover, by a result of Halberstadt and Kraus ([8], Proposition B.2.(b)), $3 \phi(N) / 200 \leq s^{n e w}(N, 2)$ for all $N \geq 61$. From this, we deduce that

$$
\frac{2 N}{(\log 2 N)^{2}} \leq \frac{2}{\log 2} \frac{\phi(N)}{\log 2 N} \leq \frac{400}{3 \log 2} \frac{s^{\text {new }}(N, 2)}{\log 2 N} .
$$


Thus, putting $k=2$ in equation (4), we get

$$
\sum_{r=1}^{d} s^{\text {new }}(N, 2)_{r} \leq 311206 d^{2} 2^{4 d^{2}}(\sqrt{p})^{d^{2}} \log p \frac{s^{\text {new }}(N, 2)}{\log 2 N} .
$$

The inequality in (5) for the case $k=2$ can be applied to prove Theorems 3 and 1 . We describe this application in the next section.

\section{Newparts of JaCOBians of MOdular CURVES}

We know that the dimension of $J_{0}^{\text {new }}(N)$ is equal to $s^{\text {new }}(N, 2)$. Also, by the work of Ribet and Shimura, the number of $\mathbb{Q}$-simple factors of $J_{0}^{\text {new }}(N)$ of dimension $d$ is equal to $s^{\text {new }}(N, k)_{d} / d$. Thus, if $J_{0}^{n e w}(N)$ is $\mathbb{Q}$-isogenous to a product of $\mathbb{Q}$-simple abelian varieties of dimension less than or equal to $d$, then

$$
s^{\text {new }}(N, 2)=\sum_{r=1}^{d} r \frac{s^{\text {new }}(N, 2)_{r}}{r} .
$$

Combining this with equation (5), we deduce that if $p$ does not divide $N$ and all $\mathbb{Q}$-simple factors of $J_{0}^{\text {new }}(N)$ are of dimension less than or equal to $d$, then

$$
s^{\text {new }}(N, 2) \leq 311206 d^{2} 2^{4 d^{2}} p^{d^{2} / 2} \log p \frac{s^{\text {new }}(N, 2)}{\log 2 N} .
$$

This proves Theorem 3 As an immediate consequence of this theorem, we observe that if $d$ is the largest dimension of the $\mathbb{Q}$-simple factors of $J_{0}^{\text {new }}(N)$, then

$$
\log 2 N \leq\left(C_{p}\right)^{d^{2}},
$$

where $C_{p}$ is a constant depending on $p$. Thus, the largest dimension of the $\mathbb{Q}$-simple factors of $J_{0}^{\text {new }}(N)$ is $\gg_{p} \sqrt{\log \log N}$. This proves a result previously obtained by Royer ([19, Theorem 1.1) using different methods. However, Royer's result was not effective. The advantage of Theorem 3 is that it explicitly shows the dependence of this implied constant on $p$. This explicit determination has an important application. We note that if all $\mathbb{Q}$-simple factors of $J_{0}^{n e w}(N)$ are elliptic curves, then taking $d=1$ in equation (6), we get that for any prime $p$ not dividing $N$,

$$
\log 2 N \leq 4979296 p^{1 / 2} \log p .
$$

How small a prime $p$ can we choose which does not divide $N$ ? If $N$ is odd, we choose $p=2$ and deduce that $\log 2 N \leq 4979296 \sqrt{2} \log 2$. If $N$ is even, the prime number theorem tells us that for a sufficiently large $N$, there is a prime $p<2 \log N$ not dividing $N$. Using effective bounds for the Chebyshev functions (see equation (5.2) in Theorem 6 of [18]), one can show that for $N>1,319,007$, there is a prime $p<1.1 \log N$ not dividing $N$. Thus, we have

$$
\frac{(\log N)^{1 / 2}}{\log \log N} \leq D
$$

for an absolute constant $D$. This proves Theorem 1. However, our bound for $N$ is clearly much larger than 1800. In future work, we would like to refine the constants in our estimates in order to yield a better bound.

We also observe that the above idea does not prove Conjecture 2 for $d>1$. This is because if we choose $p \leq 1.1 \log N$, the inequality

$$
\log 2 N \leq 311206 d^{2} 2^{4 d^{2}}(1.1 \log N)^{d^{2} / 2} \log (1.1 \log N)
$$


gives us an upper bound for $N$ only if $d^{2} / 2<1$, that is, only if $d=1$. We relegate addressing Conjecture 2 in full generality to future research.

\section{ACKNOWLEDGMENTS}

We would like to thank R. Balasubramanian, Ernst Kani, Andrew Knightly and Charles Li for their comments on a previous version of this paper. We also thank Ken Ribet for bringing Lim's work to our attention. We are also grateful to the referee for various helpful suggestions to improve the presentation of this paper.

\section{REFERENCES}

1. A. O. L. Atkin and J. Lehner, Hecke operators on $\Gamma_{0}(m)$, Math. Ann., Vol. 185 (1970), 134-160. MR0268123(42:3022)

2. M. H. Baker, E. González-Jiménez, J. González and B. Poonen, Finiteness results for modular curves of genus at least 2, Amer. J. Math., Vol. 127 (2005), 1325-1387. MR2183527 (2006i:11065)

3. D. Bressoud and S. Wagon, A course in computational number theory, Key College Publishing, Emeryville, CA, 2000. MR:1756372 (2001f:11200)

4. H. Cohen, A course in computational algebraic number theory, Graduate Texts in Mathematics, 138, Springer, 1993. MR1228206 (94i:11105)

5. H. Cohen, Sur les $N$ tels que $J_{0}(N)$ soit $\mathbb{Q}$-Isogène à un produit de courbes elliptiques, preprint, available on http://www.ufr-mi.u-bordeaux.fr/ cohen

6. L. E. Dickson, History of the theory of numbers, Vol. 3, Chelsea Publishing Co., New York, 1966. MR 0245499 (39:6807a)

7. M. Eichler, On the class number of imaginary quadratic fields and the sums of divisors of natural numbers, J. Indian Math. Soc., Vol. 19 (1955), 153-180. MR0080769 (18:299a)

8. E. Halberstadt and A. Kraus, Courbes de Fermat: résultats et problèmes, J. Reine Angew. Math., Vol. 548 (2002), 167-234. MR1915212 (2003h:11068)

9. C. Hamer, A formula for the traces of the Hecke operators on certain spaces of newforms, Arch. Math. (Basel), Vol. 70 (1998), no. 3, 204-210. MR1604056 (99b:11045)

10. M. N. Huxley, A note on polynomial congruences, Recent Progress in Analytic Number Theory, Vol. 1 (Durham, 1979), Academic Press, London-New York, 1981, 193-196. MR637347 (83e:10005)

11. A. Knightly and C. Li, Traces of Hecke operators, Mathematical Surveys and Monographs, 133, American Mathematical Society, Providence, RI, 2006. MR2273356 (2008g:11090)

12. C.-W. Lim, Decomposition of $S_{k}\left(\Gamma_{0}(N)\right)$ over $\mathbb{Q}$ and variants of partial nim, PhD thesis, University of California at Berkeley, 2005.

13. G. Martin, Dimensions of the spaces of cusp forms and newforms on $\Gamma_{0}(N)$ and $\Gamma_{1}(N)$, J. Number Theory, Vol. 112 (2005), no. 2, 197-240. MR2141534(2005m:11069)

14. M. Ram Murty, Problems in analytic number theory, Graduate Texts in Mathematics, 206, second edition, Springer, 2007. MR2376618 (2008j:11001)

15. M. Ram Murty and K. Sinha, Effective equidistribution of eigenvalues of Hecke operators, J. Number Theory, Vol. 129 (2009), no. 3, 681-714. MR2488597

16. S. Ramanujan, Highly composite numbers, Collected Papers of Srinivasa Ramanujan, AMS Chelsea Publ., Amer. Math. Soc., Providence, RI, 2000, 78-128. MR2280858

17. K. A. Ribet, Twists of modular forms and endomorphisms of abelian varieties, Math. Ann., Vol. 253 (1980), no. 1, 43-62. MR594532 (82e:10043)

18. J. B. Rosser and L. Schoenfeld, Sharper bounds for the Chebyshev functions $\theta(x)$ and $\psi(x)$, Math. Comp., Vol. 29 (1975), 243-269. MR0457373 (56:15581a)

19. E. Royer, Facteurs $\mathbb{Q}$-simples de $J_{0}(N)$ de grande dimension et de grand rang, Bull. Soc. Math. France., Vol. 128 (2000), no. 2, 219-248. MR.1772442 (2001j:11041)

20. J.-P. Serre, Répartition asymptotique des valeurs propres de l'opérateur de Hecke $T_{p}$, J. Amer. Math. Soc., Vol. 10 (1997), no. 1, 75-102. MR.1396897 (97h:11048) 
21. G. Shimura, On the factors of the Jacobian variety of a modular function field, J. Math. Soc. Japan, Vol. 25 (1973), 523-544. MR0318162 (47:6709)

22. T. Yamauchi, On $\mathbb{Q}$-simple factors of Jacobian varieties of modular curves, Yokohama Math. J., Vol. 53 (2007), no. 2, 149-160. MR2302608 (2008k:11062)

Department of Mathematics and Statistics, Queen's University, Jeffery Hall, Kingston, Ontario, Canada K7L 3N6

E-mail address: murty@mast. queensu.ca

Department of Mathematical and Statistical Sciences, University of Alberta, Edmonton, Alberta, Canada T6G 2G1

E-mail address: kaneenik@ualberta.ca 